\title{
Towards A Unified Radiology Information \& Reporting System: Challenges and Way Forwards for the Developing Countries
}

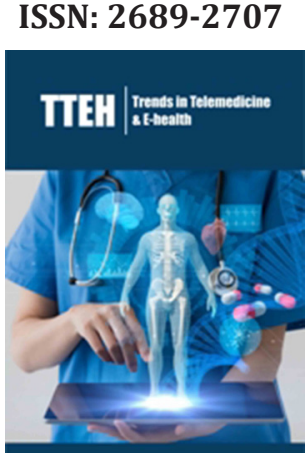

*Corresponding author: Junaid Rehman, School of Information, Systems and Modelling, Australia

\section{Submission: 眥 February 17, 2020 \\ Published: 侮 August 13, 2020}

Volume 2 - Issue 3

How to cite this article: Junaid Rehman, Marya Hameed, Ameet Kumar Jesrani, Fatuma Namisango, Zafar Mehdi. Towards A Unified Radiology Information \& Reporting System: Challenges and Way Forwards for the Developing Countries. Trends Telemed E-Health 2(3). TTEH.000540.2020. DOI: 10.31031/TTEH.2020.02.000540

Copyright@ Junaid Rehman, This article is distributed under the terms of the Creative Commons Attribution 4.0 International License, which permits unrestricted use and redistribution provided that the original author and source are credited.
Junaid Rehman ${ }^{1 *}$, Marya Hameed ${ }^{2}$, Ameet Kumar Jesrani ${ }^{3}$, Fatuma Namisango ${ }^{4}$ and Zafar $\mathrm{Mehdi}^{5}$

${ }^{1} \mathrm{PhD}$ Scholar and Casual Academic, University of Technology, Sydney, Australia

${ }^{2}$ Assistant Professor and Head of Radiology Department, NICH/JSMU, Karachi, Sindh, Pakistan

${ }^{2}$ Consultant Radiologist Cyberknife Stereotactic Radiosurgery, JPMC, Karachi, Sindh, Pakistan

${ }^{3}$ Assistant Professor, Department of Radiology, SIUT, Karachi, Sindh, Pakistan

${ }^{4} \mathrm{PhD}$ Scholar and Casual Academic, University of Technology, Sydney, Australia

${ }^{5}$ Public Health Expert and Focal Person Health Department, Govt of Sindh, Karachi, Pakistan

\section{Abstract}

A radiology report serves as an intermediary between a radiologist and referring clinician for suggesting appropriate treatment to the patients, aimed at better healthcare management. It is essentially a tool that assists radiologists in conveying their input to the patients and clinicians regarding positive or negative findings on a case. The objective of this paper is to discuss and propose Radiology Information \& Reporting System (RIRS), highlight challenges governing its implementation and suggest way forwards towards its effective implementation across the public sector tertiary care institutions of Pakistan. In the end, it is concluded that the proposed RIRS would potentially offer enormous benefits in terms of cost savings, reporting accuracy, faster processing and operational efficiency as opposed to the conventionally available manual radiology reporting procedures and systems.

Keywords: Radiology Information \& Reporting System (RIRS); Radiological Diagnostics \& Imaging; Radiology Reporting; Developing Countries

\section{Introduction}

In view of the rapid strides in the field of information systems, the radiology as a field of medical practice has witnessed ever-increasing progress in terms of improved acquisition, analysis, storage and transmission of the patient's radiological data \& information and hence supported in a more accurate, effective and faster diagnosis. This technologically assisted radiological analyses and imaging have not only augmented the productive capabilities of the radiologists and their staff but have also enhanced the overall operational efficiencies through automation of the routine radiological processes and activities. Consequently, the focus of the healthcare institutions worldwide, especially in the developed countries, has shifted towards online health information systems with an aim to effectively address issues which manual radiology reporting systems had failed to address.

In Pakistan, radiologists these days are increasingly faced with the challenges of surging costs of radiological treatment and care, consequently the demand for quality and affordable healthcare has increased. The present health information systems operating in the public sector health institutions are mostly unable to support multi-functionalities due to limited features and manual nature of processes and operations [1-3]. Currently deployed paperbased health information systems in public sector hospitals follow manual processes and require additional time to generate, process, interpret and distribute radiology reports. Because of paper-based record keeping mechanism, the process requires a lot of space and affects the cleanliness of the radiology units. Furthermore, when patient cases are written/ stored on the DVDs, these are prone to computer viruses, likely to misplace and subject to breakage. Sometimes, it also becomes very time consuming to retrieve patients' past medical records [4]. Ideally, it is expected of a professional radiologist to produce precise, consistent, and easy-to-understand reports that yield accurate results and better diagnosis \& imaging. 
However, given the dearth of healthcare facilities in the rural areas of Pakistan because of the limited staff, budget and equipment, radiologists working in the busy tertiary care centers are often faced with the challenges in terms of coordination, analysis and reporting of the radiological data and records [4,5]. Therefore, with the implementation of the proposed RIRS system, radiology units in the public sector healthcare institutions of Pakistan would be able to automate their radiological examination processes and diagnostic practices owing to improved process workflows, and systematic management \& processing of patients' clinical historical and medical records. This would support timely interventions and effective case management, thereby resulting in an efficient and quality radiology healthcare service at the reduced costs. The rest of this paper highlights as follow: The literature review gives a reasonable account of the radiological reporting standards and practices in general and is followed by the applied research methodology that leads us to proposing RIRS system. Afterwards, the hurdles to be encountered in view of the proposed system implementation are discussed. In the end, we draw upon and reflect on the advantages \& disadvantages and subsequently conclude the discussion.

\section{Theoretical Background and Literature Review}

When it comes to routine health checks, a radiological diagnosis involves examinations of unique healthcare characteristics [3].
The quality of radiological diagnosis and the accuracy of imaging reports form the essential part of a radiologist's manifestation of his healthcare expertise, contribution, and commitment towards the profession of radiology [6,7]. Nowadays, the benefits of online reporting have gained increased attention in the modern radiological practices in the developed economies. Accordingly, tele-radiology and supporting technologies have become the heart \& soul of technology-assisted healthcare practices because of their profound effect on patient's healthcare quality and improved health outcomes [4]. In this regard, a contemporary workflowenabled information system can offer much-needed radiological data automation, processing, monitoring and management functionalities, thereby offering ease of access to patient reports anywhere and anytime round the clock [8]. Additionally, it can help referring healthcare professionals, medico-legal officers, billing/ coding specialists and researchers to extract, analyze and compare key information from the radiology reports [2]. It would also enable better co-ordination between various medical wards, units, and departments in terms of exchange of radiological data, information, and records at a single click [3,4]. In view of this, Zhang et al (2009) suggests following generic work-flow-based information system of radiology. In this system, a Workflow Management System forms the core part of the system architecture that coordinates the workflows and manages each building block to control the holistic system functioning (Figure 1).

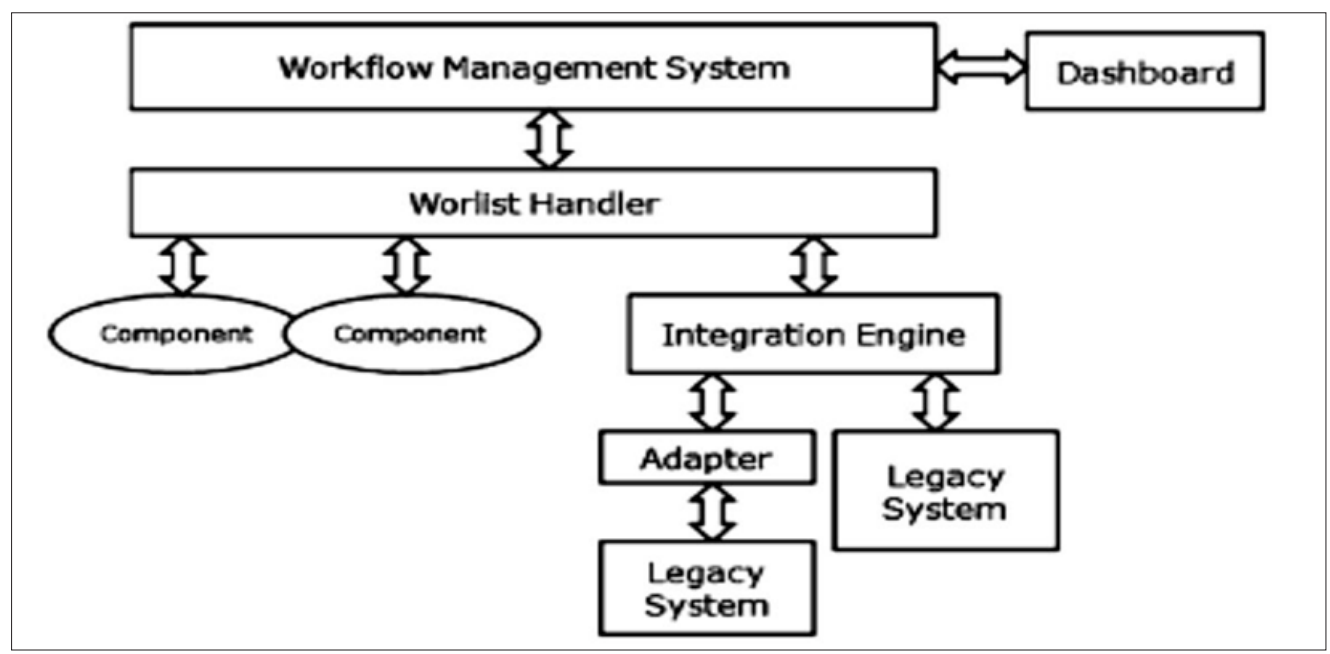

Figure 1: General Architecture of the Radiology Information System

In the developed world, a typical Radiology Information System (RIS) constitutes blend of software, systems and technologies such as DICOM (Digital Imaging and Communications in Medicine), PACS (Picture Archiving and Communication System) etc. that are used to record, process and issue patient's medical information and images such as X-ray, CT scan, MRI scan, ultrasound reports etc. Given that the data standardization is a key prerequisite for developing the PACS enabled RIS, the DICOM capabilities allow digital data integration from the video camera, scanners, printers, work-stations, servers and other networking devices and these capabilities are incorporated into one PACS. Moreover, the standardization in DICOM has supported consistency of the data and results at the time of interaction between the network equipment from different vendors that involve data storage, handling, printing, and transmission [8]. In addition to having the required technical functionalities in a standard Radiology Information System (RIS), it is also important to consider the ethical aspects underlying teleradiology activities and practices [9].

To this end, a number of researchers have emphasized on exercising ethics and fairness throughout the patient-doctor interaction. Pertinent in this regard is to consider the viewpoint of both the stakeholders i.e. radiologists and the patients. This is because some patients openly embrace technology-assisted remote healthcare while other view it as something that lack elements of 
respect and due care [5]. This could be due to emotional fear and anticipated severity of the ailment in some patients. Such patients have little or no realization of the associated cost-benefits of the healthcare technologies that are made available to them and required considerable costs in staff trainings and maintenance of the patient information systems by the healthcare providers [10]. Having these issues in mind, a radiologist's ability to effectively communicate and truly feel the pulse of the patients by being compassionate to them would go a long way. Above discussion necessitates that an holistic information system of radiology should be developed that is capable of performing the above functions along the professional lines in a manner that it is also able to maintain compatibility with the existing information systems of radiology.

\section{Research Methodology}

This research followed a qualitative case study as the methodological choice that involved personal observations by two consultant radiologists in addition to the review of 5-year patient's radiological records in three public sector hospitals/radiology \& imaging units of Karachi, Sindh, Pakistan. Besides, the researchers also accessed the government plans and reports on health sector reforms to understand non-technical (legal, political) aspects of the proposed system implementation.

\section{Proposed RIRS - Structure, Process Flow and Subsystems}

The structure of the proposed RIRS would be based on four Subsystems that are systematically interconnected in the process flow to constitute a holistic Radiology Information \& Reporting System (RIRS). These key subsystems include:

a. Patient Referral Subsystem

b. Radiological Image Acquisition \& Processing Subsystem c. Review \& Interpretation Subsystem

d. Inter-departmental Coordination \& End Reporting Subsystem

In view of the functioning of the proposed RIRS, the preliminary step that would form the key part of RIRS functioning would be to clinically review the patient referral request received from the concerned medical department/hospital. As proposed in the below diagram, the review of referred patient clinical history would serve as a basis of initial RIRS process flow. In this step, the medical imaging of each referred case would be collected at the digital workstation. In this regard, the Picture Archiving Communication System (PACS) and Digital Imaging and Communications in Medicine (DICOM) would enable digital radiological processing and interpretation of the patient's medical information. In the subsequent step, the patient reports would be thoroughly reviewed and interpreted by the examining radiologist keeping in view the medical history provided. In conjunction with this step, the process flow may also involve face-to-face or remote communications with the patients by the radiologist depending upon their physical locations. Moreover, if an additional interpretation is required, the examining radiologist may make necessary modifications in the patient medical reports. Interpretation process could also involve verbal discussion/coordination with the General Practitioners (GP) of the referring hospitals for getting further clarifications or with a peer professional radiologist with a view to seeking secondopinion. This optional process flow step would help enhance the quality of the diagnosis, thereby enabling accurate decisions and prompt release of the final case reports. The entire RIRS structure, process flow and interacting subsystems along with their building blocks are pictorially shown below (Figure 2).

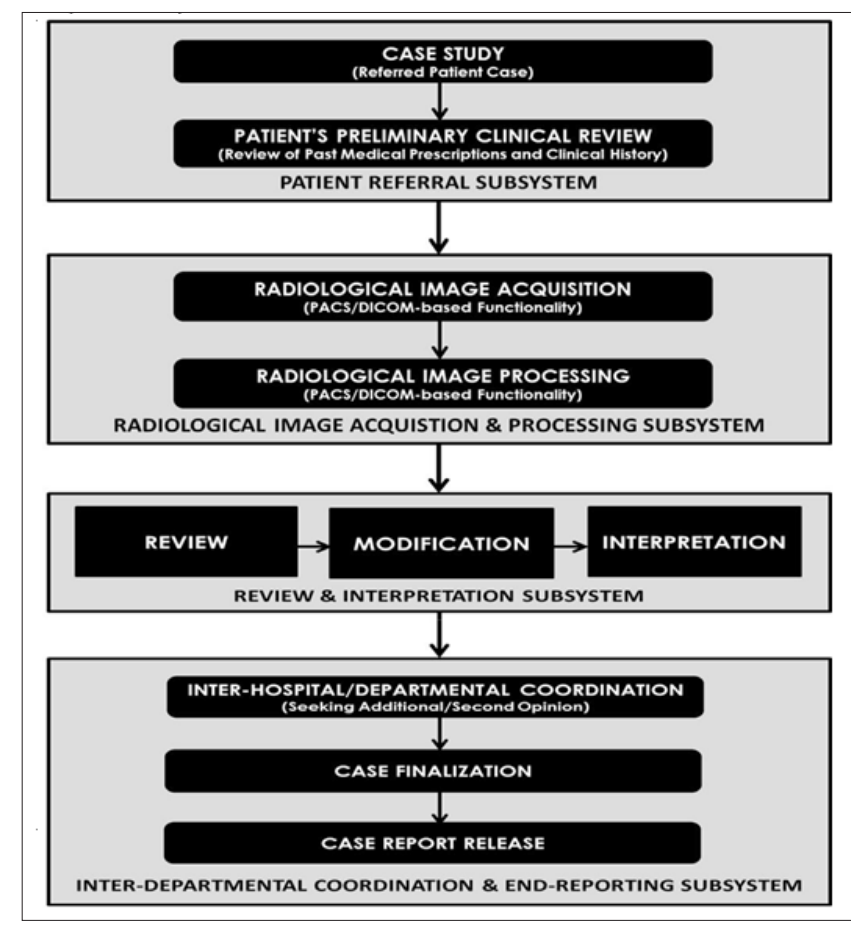

Figure 2: Proposed Radiology Information \& Reporting System (RIRS) 


\section{Challenges Governing Implementation of RIRS}

While there are certainly a number of Radiology Information Systems of rudimentary nature in use by the public and private sector hospitals in Pakistan, however, there are obvious technical and legal challenges towards the implementation of tele-radiology practices in general and proposed Radiology Information \& Reporting System (RIRS) in particular. Besides, there are also some underlying risks that could potentially hamper its integration in the public healthcare system of Pakistan.

\section{Technical/Functional Hurdles}

a. When it comes to transmission of the images, there are issues like variation in registration numbers in addition to other security concerns affecting the integrity of data and resulting in an images being received which may not be the true version of the images actually transmitted [2].

b. Although PACS/DICOM could improve the productivity and efficiency of the healthcare system, however, this being digital system in nature, its deployment would attract huge investment mainly in the rural settings as currently analog systems are being used in rural hospitals. This makes its country-wide implementation quite expensive.

\section{Legal/Statutory Hurdles}

a. After the advent of 18th amendment in the 1973 constitution of Pakistan, health was made provincial (state) subject, empowering provinces to develop, review and legislate their own health related policies and matters independently. Neither any province (state) nor the federal government could intervene in the health matters of other province (state) [10]. Hence any such proposals for country-wide development of a unified Radiology Information \& Reporting System would require enunciation through the Council of Common Interest (CCI) after broad consensus of all the states/provinces.

b. In the wake of provincial autonomy as enshrined in the 18th constitutional amendment in 1973 constitution of Pakistan, every province (state) has a right to deploy any such online reporting systems, this could however, result in an incompatible/ inconsistent reporting systems when it comes to the mobility of the patient records and imaging within the provinces, calling for a need of a unified and consensus based online reporting system.

\section{Advantages of the Proposed RIRS}

a. While there are potential hurdles behind the implementation of the proposed RIRS and these must be removed to reap the true benefits of tele-radiology concepts and practices in Pakistan. However, if implemented, it would offer following advantages.

b. This proposed RIRS would enable speedy acquisition, interpretation, and digital processing of the patient's data, thereby enabling the busy radiologists to timely share diagnostic findings all using an integrated system. c. The proposed online image processing and reporting system effectively serves the purposes like image manipulation and navigation between the image-sets owing to incorporation of PACS based system capabilities.

d. Yet another merit of RIRS is that the access to patient's medical data provided to the radiologists would not be just limited to the hospitals but also beyond normal working hours. Communication with the radiologist could be maintained anytime, anywhere and even outside the hospitals/diagnostic centers. This would particularly benefit the patients requiring emergency treatment.

e. In rural areas of Pakistan, the access to highly competent and qualified radiologist is limited. Hence the implementation of proposed system would specifically be beneficial for the people living in the remote/far-flung areas of the country and they would be able to avail the services of the experienced and technicallycompetent radiologists who are usually based in the metropolitan cities of the country.

f. Patient medical history and clinical information form the key part of radiological diagnosis, enabling the radiologists to come up with meaningful and accurate diagnosis. Most often, a limited medical history of the patients is made available to the radiologists and disease symptoms are poorly narrated by the patients, sometimes in two words format like headache, abdominal pain etc.). This does not always help radiologists to pinpoint the exact diagnosis of the disease. However, the presence of an online reporting system would eliminate this issue.

g. In case of the patients who often do not maintain, misplace or keep a track of their past medical records, it sometimes becomes difficult for a radiologist to ascertain the changes in the images and films as compared to the past. Given the proposed systematic capabilities of RIRS to archive and store patient's past medical records, the online reporting functionalities would help archive all past medical records and imaging at one place which can be easily accessed by the radiologists, resulting in saving time and accurate diagnosis.

h. In case of manual reports, hardcopy of the reports and records are difficult to manage and there is a likelihood of these being misplaced. However, online access to health records by the virtue of RIRS would enable patients to stay updated on their current health status, thereby enabling them to access their reports anytime and anywhere to make more informed decisions on their healthcare status.

i. Online access to records allows patients to seek additional opinion from other consultants electronically. This would provide patients with new options to make better decisions and seek best possible healthcare at economical rates under given circumstances.

j. Patient's radiology-related statistical data would help monitor and evaluate the performance appraisal of the professional staff involved in undertaking radiological services in the tertiary healthcare establishments. 
k. Last but not the least, while the proposed online RIRS would envisage substantial capital investment, nevertheless, the potential benefits to be realized would far exceed the demerits of the system.

\section{Limitations}

a. At one end, the proposed radiology reporting system would offer range of benefits, this would, however, come at the expense of assumingly following limitations.

b. Patients, while enjoying the benefits of tele-radiology, may come across the concerns on the privacy of their medical records in terms of these being hacked or the data be leaked to the marketing companies. This might end-up make some patients reluctant to disclose key information which can affect the quality of their diagnoses and treatments.

c. While becoming a part of RIRS, patients would be expected to make computer-based online appointments, receive health information, and interact with other healthcare professionals. The patients might want to prefer face-to-face interaction instead of online.

d. Despite the several advancements made in digital data/ image processing, yet a clinical weakness of tele-radiology is its inability to integrate processed images that belong to or are part of any other radiology/healthcare information system i.e. in an instance when the radiological data are transmitted between two or more hospitals/healthcare institutions employing incompatible radiology information systems.

e. There is also no direct access for interpreting radiologist at the receiving station to acquire additional real-time imaging data available at the sending site repositories due to being accessed from a remote location. Whereas, in the case of conventional film-based radiology reporting systems, additional information like intradepartmental reviews, references and consensus of experts are available in a real-time manner for cross-verification and correct examination of the reports.

f. Yet another bottleneck when it comes to the implementation of online RIRS, particularly in the rural areas, is that ultrasound is the only tool conventionally available for radiological investigations and quick diagnosis in the real-time manner while the existing system's inability to provide real-time imaging information could result in a possibility of incorrect diagnosis.

g. A small delay in eliminating the possible problems caused due to the failure of PACS/DICOM based functionalities could endup in upsetting consequences.

h. Once the proposed system is deployed and the moment hospitals/medical institutions go filmless, the resources to produce, view, interpret, store and transmit films won't exist anymore.

\section{Discussion and Implications}

This paper presents the idea of introducing a Radiology Information \& Reporting System (RIRS) in the public sector tertiary care institutions of Pakistan. While not always achievable, nevertheless, we do provide food for thought to the health ministries, departments, authorities, and the professionals involved in the radiology \& diagnostics practices in the healthcare institutions of Pakistan. Incorporation of the proposed RIRS system would help revamp the workflow processes of the radiologists by offering ease of patient records management and digital data processing, enabling accurate and faster reporting services. However, it is again reiterated to exercise and incorporate the element of beneficence when deploying online healthcare systems and technologies as the healthcare providers might be comfortable with the use of telehealth technologies but the patients might have concerns on the security, privacy and affordability of the electronic health systems [9-12]. These aspects of beneficence, respect and fair treatment should form mandatory part of the institutional care procedures \& policies, and staff should adhere to these when working on and using these technologies to avoid potential harm to the patients. It would also be in the fitness of things that the healthcare staff giving care to patients should also be treated with equal fairness and respect by their employers. These principles of fairness and credibility would be crucial towards the success of digital healthcare initiatives in general and online health information systems. Yet another aspect ensuring fairness of digital healthcare initiatives is to build and maintain a good reputation among the care-seeking individuals and communities, as the word-of-mouth and prestige are essential ingredients towards the successful launch of an online healthcare system. Accordingly, consideration of ethical aspects would effectively serve the spirit of radiological healthcare objectives. While the organizations might have to bear the costs of ensuring fairness in their healthcare practices, the benefits of maintaining an outstanding image would outweigh that much of capitalization.

On a socio-political front, it is equally important for all the federating units (provinces) to sit together, build trust and establish broad consensus on the uniform implementation of the proposed RIRS. On emotional front, while at one hand, these online healthcare technologies can enhance quality of life through better healthcare and preventive outcomes, however, it can also affect the spirit of healthcare in many ways as it's primarily about human health conditions and sentiments which necessitate deeper understanding of the patient's emotional states of mind. Last but not the least, it is critical to understand that the element of fairness, respect, accuracy, reliability, and affordability must constitute core part of digital healthcare practices in general and tele-radiology care in particular.

\section{Conclusion and Directions for Future}

The findings of this case study support the compelling need to take such digital initiatives in view of the better healthcare management and given that there is a dearth of skilled staff, budget, and equipment. While, there are always pros and cons when it comes to use of the emerging state-of-the-art systems \& technologies, nevertheless, these days the concept of utilizing information systems of radiology is increasingly gaining traction in the developing countries in all aspects of radiological diagnostics 
and imaging practices, in particular, in the remote provision of healthcare services. Hence, the need of the time is to assess whether the benefits offered by the proposed system outweigh its limitations. Given its exciting potential, we have high hopes that a judicious and responsible implementation of RIRS countrywide would benefit both the patients and healthcare institutions. Overall, this paper serves as a starting point towards radiological care and digital health management. As the scope of proposed RIRS extends within the country-wide public sector hospitals, it might require more empirical research and investigations for more effective implementation.

\section{References}

1. European Society of Radiology (2018) ESR paper on structured reporting in radiology. Insights into Imaging 9(1): 1-7

2. Gul P, Gul P (2019) Structured reporting in radiology. Are we ready to implement it? Pakistan Journal of Radiology 29(1).

3. Zhang J, Lu X, Nie H, Huang Z, Van der Aalst WMP, et al. (2009) Radiology information system: A workflow-based approach. Int J Comput Assist Radiol Surg 4(5): 509-516.

4. Munteh PA (2019) Implementation of an electronic-based health information system maintaining the paper based system-including:
A situational analysis in Banso Baptist Hospital (BBH) in Cameroon. Trends Telemed E-Health 2(1): 1-3.

5. Kenneth CL, Jane PL (2010) Management information systems $\left(12^{\text {th }}\right.$ edn), Pearson Education, USA.

6. Pool F, Goergen S (2010) Quality of the written radiology report: A review of the literature. Journal of American College of Radiology 7(8): 634-643.

7. Mityul MI, Gilcrease G, Mangano MD, Demertzis JL, Gunn AJ, et al. (2018) Radiology reporting: Current practices and an introduction to patient-centered opportunities for improvement. American Journal of Roentgenology 210: 376-385.

8. Schneider A, Feussner H (2017) Health Informatics/Health Information Technology. Biomedical Engineering in Gastrointestinal Surgery pp. 473-489.

9. Eileen EM (2020) Telemedicine/E-health and the soul of medical practice. Trends Telemed E-Health 2(2): 1-2.

10. Nishtar S (2014) Health and the 18th Amendment retaining national functions in devolution.

11. Ganeshan D, Duong PA, Probyn L, Lenchik L, McArthur TA, et al. (2018) Structured reporting in radiology. Academic Radiology 25(1): 66-73.

12. Cramer JA, Eisenmenger LB, Pierson NS, Dhatt HS, Heilbrun ME, et al. (2014) Structured and templated reporting: An overview. Applied Radiology 43(8): 18-21. 